\title{
détermination des propriétés élastiques à grande échelle d'un massif rocheux
}

\author{
determination of large scale elastic properties \\ of a rock mass
}

\author{
E. HETUIN, P. BEREST \\ Laboratoire de Mécanique des Solides*, \\ Laboratoire Commun Ecole Polytechnique, \\ Ecole des Mines, ENPC, URA 317 CNRS \\ P.A. BLUM \\ Institut de Physique du Globe \\ Université Pierre et Marie Curie ${ }^{*}$
}

Rev. Franç. Géotech. n 48, pp. 65-71 (juillet 1989)

\section{Résumé}

En vue de déterminer le module élastique moyen de masses rocheuses à grande échelle, on a mesuré au moyen d'un inclinomètre à haute résolution, les inclinaisons de la surface du sol engendrées d'une part, par la décompression d'une cavité profonde, d'autre part, par l'application d'une force ponctuelle appliquée à quelques mètres de l'inclinomètre. Le premier essai donne des résultats qualitativement intéressants; le second paraît constituer le fondement d'une méthode efficace et commode de mesure en place du module élastique d'un sol.

\footnotetext{
Abstract

In order to evaluate the large scale elastic modulus of a rock mass, dips at ground level have been measured by means of a high resolution tilmeter. The mechanical loading was, respectively, depressurization of a deep salt cavern and a vertical load applied at short distance from the tiltmeter. The first test gives satisfactory qualitative results; the second test provides an easy and efficient method for evaluation of the in situ elastic modulus of soils at ground level.
} 


\section{NOTATIONS}

E

$\mathrm{F}$

$\mathrm{H} \quad$ Profondeur du centre de la caverne

p Variation de pression dans la caverne

$r_{0} \quad$ Rayon de la caverne

$r$ Distance horizontale à l'inclinomètre

$\mathrm{U}_{2} \quad$ Déplacement vertical

$x, y, z$ Coordonnées cartésiennes

$\lambda, \mu \quad$ Coefficients élastiques de Lamé

$\nu \quad$ Coefficient de Poisson

$\pi \quad \mathrm{Pi}$

$\rho \quad$ Distance à l'inclinomètre

\section{INTRODUCTION}

Il est classique d'observer qu'un effet d'échelle affecte la plupart des grandeurs mesurables en géotechnique. Par exemple, la perméabilité d'une roche peut être mesurée sur éprouvettes, au laboratoire; ou bien dans des sondages in situ; parfois à l'échelle du massif lorsqu'un bilan des débits entrant et sortant est possible. Entre ces trois mesures, des écarts de plusieurs puissances de dix sont possibles (W.F. BRACE, 1980).

De même pour les grandeurs mécaniques, même les plus courantes (module d'élasticité), un écart important est fréquement observé entre les résultats d'essais de laboratoire et ceux de mesures in situ intéressant un plus grand volume de roches (la convergence naturelle d'une galerie, par exemple). On peut penser qu'un facteur d'échelle plus important apparaîtrait pour des mesures concernant un très grand volume de roches (de l'ordre du kilomètre cube) mais il est exceptionnel qu'on puisse évaluer la réponse d'un massif entier; il faut pour cela que la sollicitation mécanique soit à l'échelle du massif. La mise en eau d'un barrage constitue un exemple d'une telle sollicitation (P. EGGER, L. MULLER, 1982).

On propose dans la présente note d'évaluer les propriétés mécaniques d'un massif à deux échelles: celle des terrains de surface (les premiers mètres), et celle de l'ensemble du massif (quelques centaines de mètres d'épaisseur), sous l'effet d'un chargement modéré, au moyen d'un appareil de mesure extrêmement sensible, l'inclinomètre de l'Institut de Physique du Globe de Paris (IPGP).

Les essais correspondants ont pu être conduits grâce à l'efficace collaboration du Gaz de France (Département des Réservoirs Souterrains et Exploitation d'Etrez).

\section{APPAREILLAGE}

L'inclinomètre est constitué d'un équipage mobile autour d'un axe (J.P. ASTE et al., 1986, P.A. BLUM, 1963, P.A. BLUM et al., 1971) et dont le centre de gravité est excentré par rapport à cet axe.
Si celui-ci est parfaitement vertical, l'équilibre est indifférent. $\mathrm{Si}$ l'axe présente une certaine inclinaison par rapport à la verticale, le centre de gravité de l'équipage mobile se placera dans le plan commun à laxe de rotation et à la verticale. Un système optique permet de repérer la position de ce plan, et donc les variations de l'inclinaison.

La particularité de l'inclinomètre est d'être constitué d'un monobloc de silice, ce qui évite tout frottement solide et réduit très sensiblement les perturbations d'ordre thermique. Dans sa version géotechnique, la sensibilité de l'appareil est inférieure à $10^{-8}$ radian (soit une pente de $1 \mathrm{~mm}$ pour $100 \mathrm{~km}$ ). Toutefois, cette sensibilité théorique doit être réévaluée dans le contexte concret de chaque essai particulier. Comme on le verra, la dérive naturelle du site où sont installés les appareils, l'activité microsismique ambiante, l'influence des variations diurnes de température, conduisent à choisir de détecter des phénomènes suffisamment brefs.

\section{DISPOSITIF DE MESURE}

L'objectif de l'essai étant à l'origine de mesurer les effets induits en surface par une variation de la pression dans une cavité, nous avons disposé l'instrumentation au point qui semblait optimal pour obtenir le meilleur signal (cf. fig. 1). Les calculs ont montré que pour une cavité de $230000 \mathrm{~m}^{3}$ située à $1300 \mathrm{~m}$ de profondeur, ce point doit se trouver à environ $650 \mathrm{~m}$ de la tête de puits.

Nous avons donc installé 3 plots de mesure, chacun contenant une paire d'inclinomètres orientés, l'un dans la direction de la cavité, l'autre dans une direction perpendiculaire, ce pour des raisons qui apparaîtront plus loin. Etant donné l'extrême sensibilité des appareils aux variations thermiques, chaque plot était soigneu-

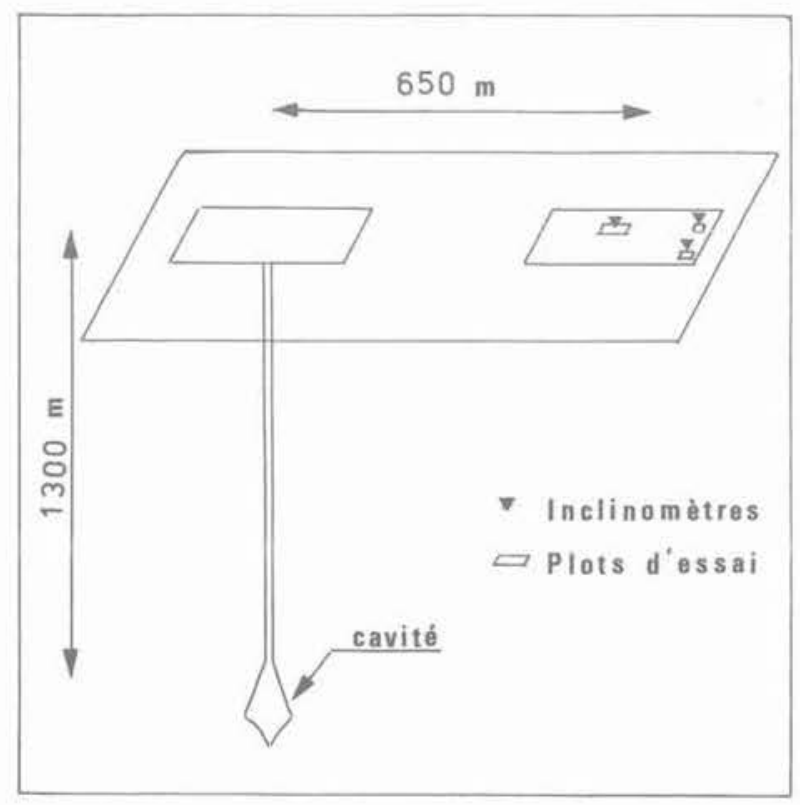

Fig. 1. - Dispositif de I'essai.

Fig. 1. - Schematic view of the test. 
sement isolé et équipé d'une sonde de température afin de pouvoir ensuite séparer la dérive d'origine thermique du signal utile.

Les trois plots étaient de conception différente pour pouvoir examiner l'influence de la nature de la liaison entre le support de l'inclinomètre et le terrain. Notons d'abord que, au moins dans l'hypothèse élastique, et pour un système de coordonnées cylindriques d'axe vertical passant par le point d'application de la sollicitation, les directions verticale, radiale, et orthoradiale sont principales pour la déformation à la surface du sol, de sorte que ces directions y subissent une rotation d'ensemble sans distorsion. Tous les modèles de fixation de l'inclinomètre au terrain sont donc en principe équivalents.

Le plot $n^{\circ} 1$ (cf. fig. 2) est constitué d'une excavation $\left(2 \times 2 \times 2 \mathrm{~m}^{3}\right)$ dont les parois verticales sont bétonnées. Au fond de cette excavation se trouve une petite dalle de béton $\left(0,6 \times 0,6 \times 0,3 \mathrm{~m}^{3}\right)$ solidaire d'un pieu métallique de $5 \mathrm{~m}$ de long foncé dans le sol. C'est sur cette dalle qu'est installée l'instrumentation, le pieu étant là pour assurer un bon couplage avec le terrain naturel. Pour des raisons de protection thermique, les parois de l'excavation sont revêtues de polystyrène (10 cm d'épaisseur), l'ensemble étant recouvert d'un capot lui aussi doublé d'isolant.

Le plot $n^{\circ} 2$ est identique au précédent à ceci près qu'il ne s'y trouve pas de pieu.

Le plot $n^{\circ} 3$ est constitué par une cave bétonnée de $4 \mathrm{~m}$ de fond sur environ $4 \times 2 \mathrm{~m}^{2}$ de large, qui

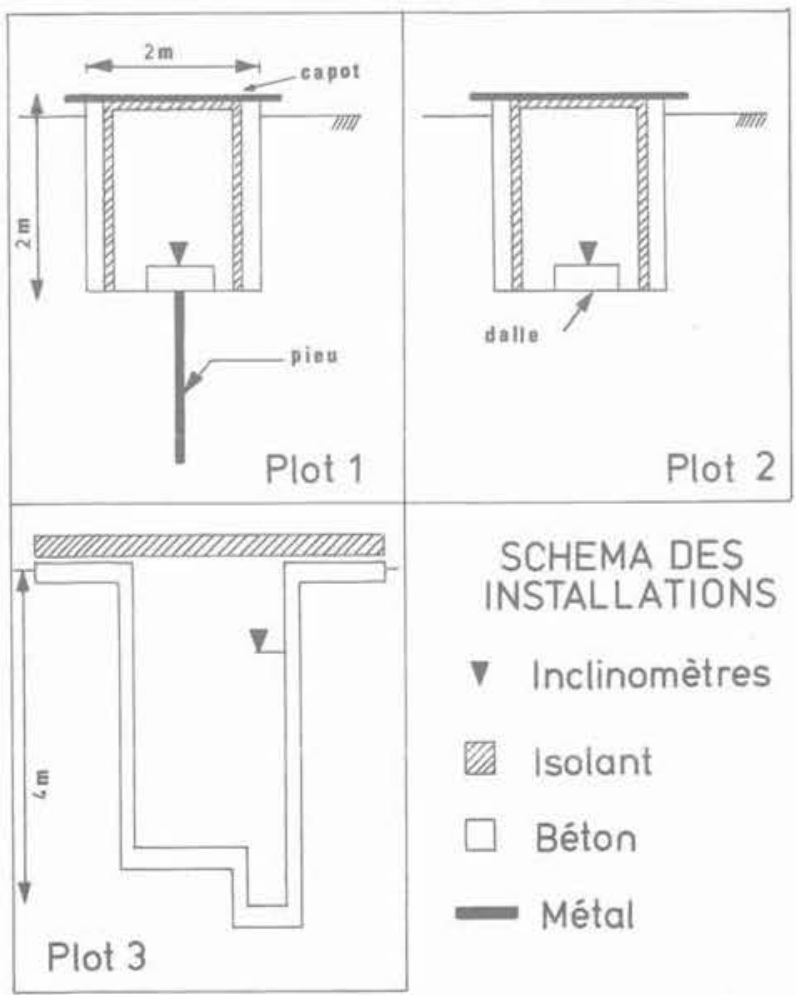

Fig. 2. - Coupe verticale des plots d'essai.

Fig. 2. - Vertical cross section of the tiltmeter emplacements. abrite la tête d'un puits foré en 1985 et à partir duquel sera ultérieurement creusée une cavité de stockage. Au fond de cette cave se trouve un puisard où plonge une pompe flottante chargée d'évacuer l'eau en cas de pluies. Dans ce plot, les inclinomètres furent installés sur les parois verticales. De même que pour les dispositifs précédents, l'ensemble a été revêtu d'isolant.

Les signaux issus des appareils et des sondes de température étaient enregistrés simultanément de façon numérique et analogique pour nous permettre un suivi en temps réel de l'essai et un important traitement ultérieur de l'information (décorrélation du signal par la température, filtrage de l'activité microsismique).

\section{DÉTERMINATION D'UN MODULE ÉLASTIQUE DE SURFACE}

L'inclinomètre de lIPGP possède une direction privilégiée de sensibilité; le signal obtenu sur l'appareil est donc la projection de l'inclinaison vraie sur cet axe. Aussi nous a-t-il paru nécessaire, préalablement à toute mesure, de déterminer aussi précisément que possible cette direction de sensibilité. Pour ce faire, nous avons placé successivement la même charge en plusieurs points autour du plot de mesure (cf. fig. 3) de façon à décrire un cercle. Les signaux issus des deux appareils situés dans ce plot ont été enregistrés (cf. fig. 4). Les inclinomètres sont grossièrement perpendiculaires et il apparait clairement sur la figure, que le signal est maximal sur un appareil quand il est nul sur l'autre. Nous avons dès lors pu sélectionner une direction privilégiée qui a ensuite été l'axe suivant lequel nous avons effectué un profil pour estimer le module d'élasticité des terrains de surface.

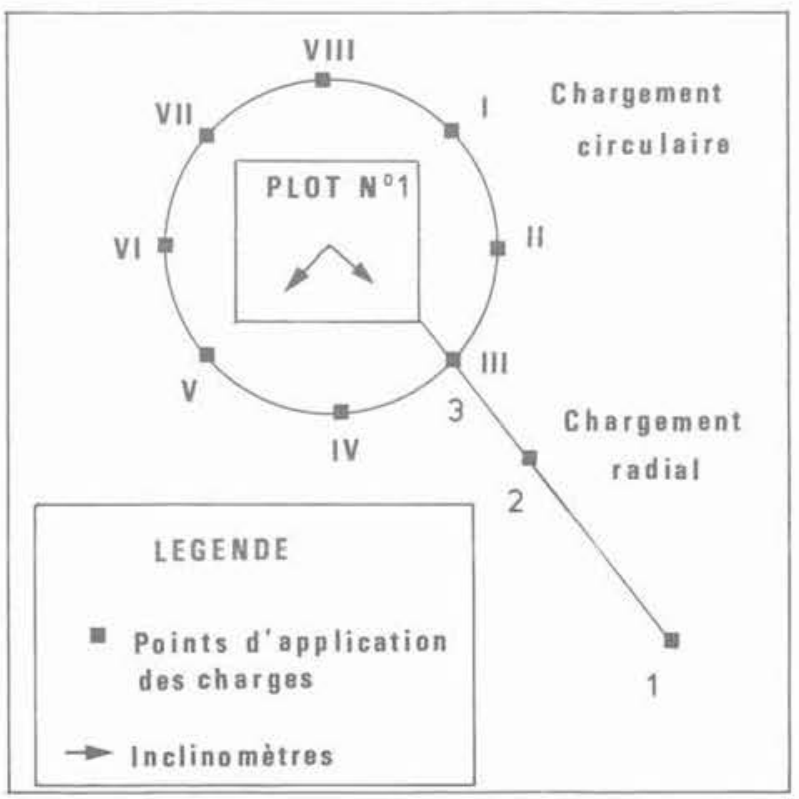

Fig. 3. - Chargements circulaire et radial. Fig. 3. - Circumferential and radial loadings. 


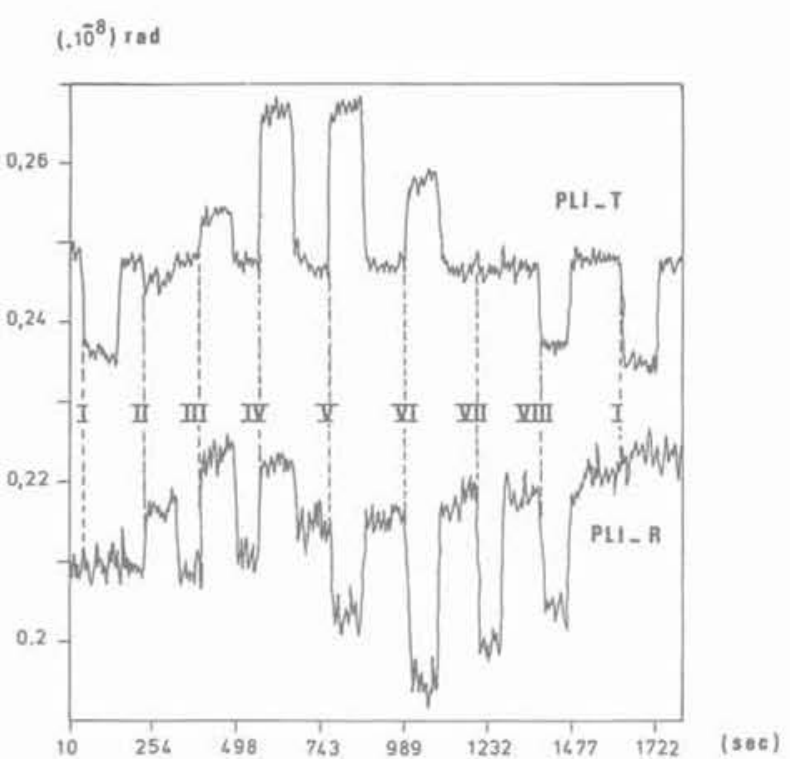

Fig. 4. - Inclinaisons observées lors du chargement circulaire. Fig. 4. - Observed dips during the circumferential loading.

Une force verticale (poids d'une automobile) a alors été appliquée successivement à des distances différentes du plot de mesure; l'inclinaison était enregistrée de façon continue pour pouvoir observer la charge (points 1, 2 et 3 sur la figure 5) et la décharge (points $\mathrm{A}, \mathrm{B}$ et $\mathrm{C}$ ). On constate que, pour une distance donnée, l'amplitude des signaux induits par la charge et la décharge est identique, ce qui indique que le système a une réponse élastique.

Une fois connue l'inclinaison (fonction de la distance d'application de F), nous avons cherché à détermi-

\section{$\left(.10^{-7}\right) \mathrm{rad}$}

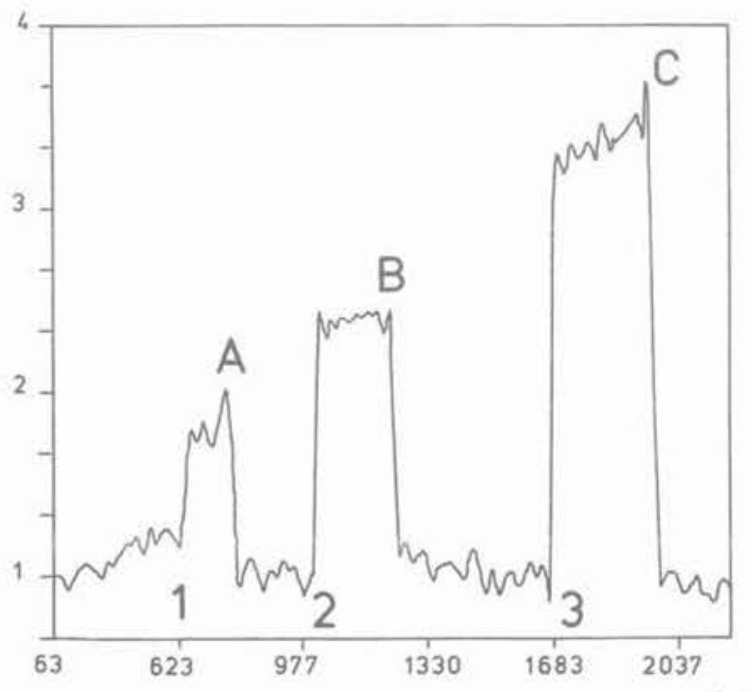

( $\sec$ )

Fig. 5. - Inclinaisons observées lors du chargement radial (plot 1).

Fig. 5. - Observed dips during the radial loading (emplacement 1). ner le module d'Young des terrains de surface. Pour cela, nous avons utilisé la solution classique de Boussinesq (J. MANDEL, 1966) qui donne l'effet d'une charge ponctuelle appliquée à la frontière d'un espace semi-infini élastique. Le système de coordonnées utilisé est décrit sur la figure 6 .

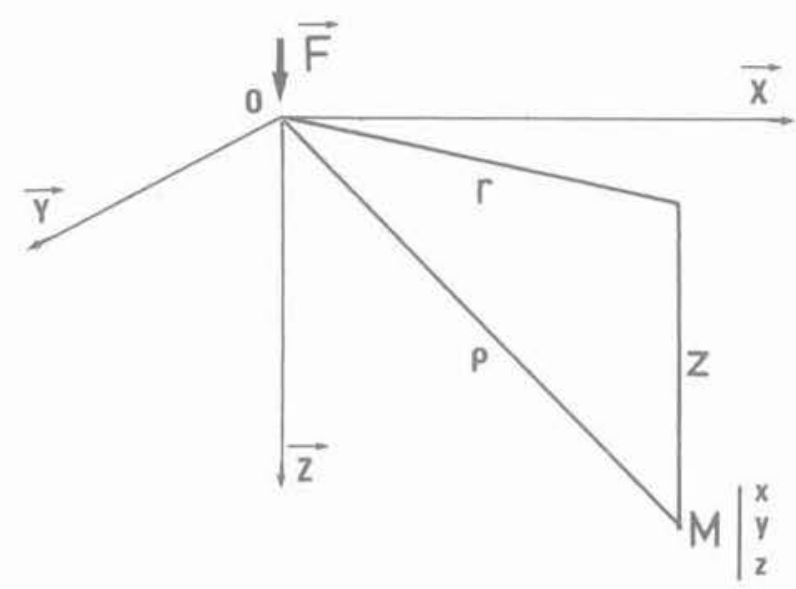

Fig. 6. - Système de coordonnées.

Fig. 6. - Spherical and cylindrical coordinates.

On a :

$\mathrm{U}_{\mathrm{z}}=[\mathrm{F} /(4 \pi \mu \rho)]\left[(\lambda+2 \mu) /(\lambda+\mu)+z^{2} / \rho^{2}\right]$

où $\rho=\left(r^{2}+z^{2}\right)^{1 / 2}$

D'où :

$$
\begin{gathered}
\partial \mathrm{U}_{\mathrm{z}} / \partial \mathrm{r}=\left[-\mathrm{Fr} /\left(4 \pi \mu \rho^{3}\right)\right] \\
{\left[(\lambda+2 \mu) /(\lambda+\mu)+3 \mathrm{z}^{2} / \rho^{2}\right]}
\end{gathered}
$$

avec :

$$
\lambda=E \nu /[(1+\nu)(1-2 \nu)]
$$

et :

$$
\mu=\mathrm{E} /[2(1+\nu)],
$$

il vient :

$$
\begin{gathered}
\partial \mathrm{U}_{z} / \partial \mathrm{r}=\left[-(1+\nu) \mathrm{Fr} /\left(2 \pi \mathrm{E} \rho^{3}\right)\right] \\
{\left[2(1-\nu)+3 z^{2} / \rho^{2}\right]}
\end{gathered}
$$

Le poids de l'automobile est de $\mathrm{F}=7500 \mathrm{~N}$ et la profondeur de l'inclinomètre est environ $\mathrm{z}=2 \mathrm{~m}$. En labsence de mesure précise, on a retenu $\nu=0,3$.

Nous avons les données mesurées suivantes, qui sont déterminées avec une précision de $0,05,10^{-7} 7$ radian:

$$
\begin{aligned}
\text { Pour } r & =10 \mathrm{~m}, \quad\left|\frac{\partial \mathrm{U}_{z}}{\partial \mathrm{r}}\right|=0,9 \cdot 10^{-7} \mathrm{rad} \\
\mathrm{r} & =6,6 \mathrm{~m},\left|\frac{\partial \mathrm{U}_{z}}{\partial \mathrm{r}}\right|=2 \cdot 10^{-7} \mathrm{rad} \\
\mathrm{r} & =4,6 \mathrm{~m},\left|\frac{\partial \mathrm{U}_{2}}{\partial \mathrm{r}}\right|=3,5 \cdot 10^{-7} \mathrm{rad}
\end{aligned}
$$


On obtient donc:

$$
\text { pour } \begin{aligned}
\mathrm{r} & =10 \mathrm{~m}, \mathrm{E}=250 \mathrm{MPa} \\
\mathrm{r} & =6,6 \mathrm{~m}, \mathrm{E}=260 \mathrm{MPa} \\
\mathrm{r} & =4,6 \mathrm{~m}, \mathrm{E}=300 \mathrm{MPa}
\end{aligned}
$$

Il est satisfaisant que l'on obtienne, pour les trois mesures, un module relativement peu dispersé. On doit tenir compte des éléments suivants:

- la précision de $0,05 \cdot 10^{-7}$ radian sur les données mesurées induit une incertitude de quelques pourcent pour le module $\mathrm{E}$;

- la solution de Boussinesq constitue une approximation; d'une part la force "ponctuelle» est en fait transmise par les quatre roues du véhicule; d'autre part, la réponse du système cave-dalle de béton-pieu (s'il y a lieu) peut s'écarter de la réponse affectant théoriquement la direction verticale en un point du voisinage de la dalle;

- la distance exacte d'application de la force «ponctuelle» est nécessairement affectée d'une incertitude, et le coefficient de Poisson n'est qu'estimé;

- enfin, le module mesuré est une certaine moyenne des modules vrais du terrain, certainement inhomogène verticalement; cette moyenne n'est pas la même à toute distance de l'inclinomètre; l'influence des terrains les plus superficiels est plus grande à courte distance. Ainsi, sous la réserve d'une estimation plus précise de l'incertitude, il semble que le module croisse lorsqu'on se rapproche de l'inclinomètre. On peut penser qu'alors le remblai compacté de faible épaisseur, probablement plus raide que les terrains sous jacents argilo-sableux, a une influence plus importante dans le module global mesuré.

Cette première expérience a également permis de comparer les dispositifs retenus pour chacun des plots. Les enregistrements effectués sur le plot n ${ }^{\circ} 2$ (sans pieu) sont représentés sur la figure 7: on note que les appareils enregistrent la charge, mais non la décharge. Par la suite, d'ailleurs, ce plot a présenté un comportement assez erratique. On peut aussi penser que sur le plot $n^{\circ} 1$ (avec pieu), c'est le pieu, solidaire des terrains plus profonds moins sollicités par le chargement, qui assure en partie le retour élastique de la dalle dans sa position d'équilibre initiale.

\section{DÉTERMINATION D'UN MODULE D'ÉLASTICITÉ GLOBAL}

L'essai consiste à faire varier la pression d'une cavité souterraine remplie de saumure de $230000 \mathrm{~m}^{3}$ de volume, située à une profondeur de 1300 mètres environ dans le massif salifère du gisement de Bresse.

On s'efforce de mesurer au moyen du réseau d'inclinomètres de surface décrit plus haut la déformée de la surface induite par ce chargement.

Les dimensions de la cavité autorisent à assimiler celle-ci à une sphère dans un demi espace que l'on suppose élastique linéaire homogène et isotrope. La

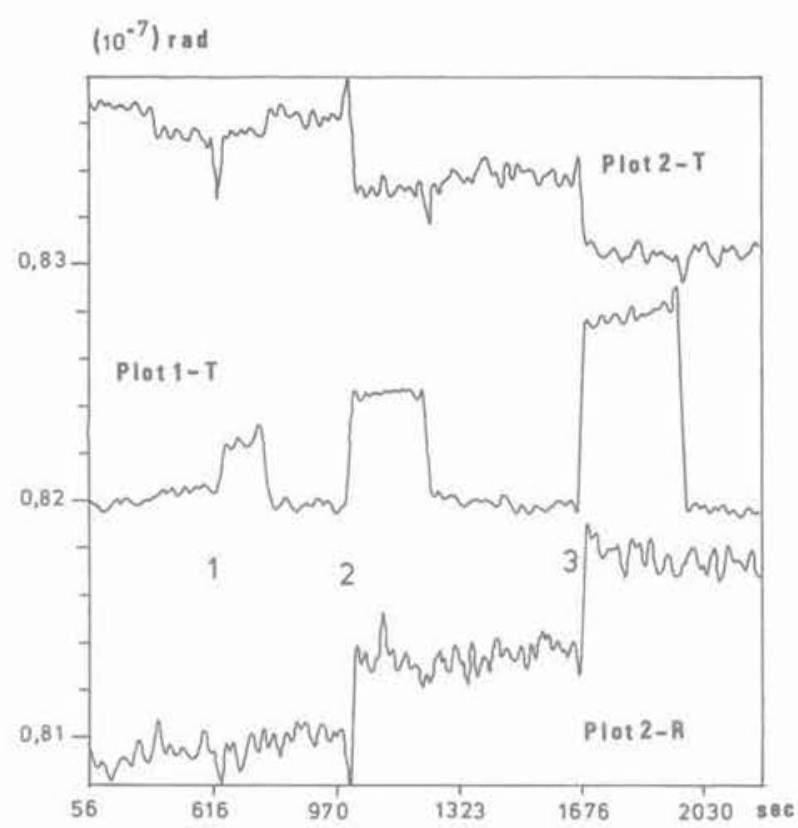

Fig. 7. - Inclinaisons observées lors du chargement radial (plots 1 et 2).

Fig. 7. - Observed dips during the radial loading lemplacements 1 and 2).

solution exacte de ce problème peut être obtenue $(E$. HETUIN, 1988); pour la simplicité de l'exposé, on se contentera ici d'une solution approchée dans laquelle la cavité est assimilée à un point, $\mathrm{p}$ désignant toutefois la pression vraie appliquée et $\mathrm{r}_{0}$ le rayon d'une sphère de même volume que la cavité réelle. $\mathrm{H}$ désignant la profondeur de la cavité et $\mathrm{r}$ la distance horizontale à la tête de puits, l'inclinaison théorique en un point de la surface du sol est alors:

$$
\begin{gathered}
\partial \mathrm{U}_{2} / \partial \mathrm{r}=3\left[\left(\mathrm{p} \mathrm{r}_{0}^{3}\right)(1-\nu) / \mu\right] \\
{\left[\mathrm{Hr} /\left(\mathrm{H}^{2}+\mathrm{r}^{2}\right)^{5 / 2}\right]}
\end{gathered}
$$

Le maximum d'inclinaison est obtenu au point d'inflexion de la déformée (cf. fig. 9) soit pour $r=\frac{H}{2}$, ce qui explique que les dispositifs de mesure aient été placés à 650 mètres de la tête de puits de la cavité dont on fait varier la pression interne. Cette inclinaison reste néanmoins faible, de l'ordre de $10^{-8}$ radian pour une valeur vraisemblable du rapport $\mathrm{p} / \mu$ (quelques millièmes), ce qui soulève diverses difficultés:

- la direction du maximum d'inclinaison est délicate à déterminer a priori sur le terrain, ce qui a conduit à disposer deux inclinomètres suivant des directions orthogonales dans chaque plot;

- les variations diurnes de la température atmosphérique engendrent des dilatations des terrains de surface dont les effets peuvent être supérieurs à ceux que l'on souhaite enregistrer. La température est donc enregistrée dans chacun des plots de mesure; on a pu mettre en évidence une très bonne corrélation entre son évolution et les variations naturelles d'inclinaison enregistrées par l'inclinomètre, ce qui permet de séparer les diverses influences dans le signal obtenu. De 
plus, on a profité de conditions météorologiques assez favorables (temps humide assurant une bonne stabilité thermique). Néanmoins, les perturbations apportées par la température apparaissent, comme dans beaucoup de mesures fines, un facteur essentiel.

On peut contourner cette dernière difficulté en effectuant des variations de pression intenses et brèves dans la cavité. Les contraintes techniques d'injection interdisent toutefois d'accroître de $5 \mathrm{MPa}$ la pression d'une cavité en moins de quelques heures; aussi estce plutôt le début de la décompression, beaucoup plus rapide, sur lequel un espoir d'enregistrement d'un signal net s'est porté.

Cing cycles de compression/décompression, d'une durée totale de six heures chacun, ont été effectués, à raison d'un cycle par jour. Les succès ont été variables; on présente sur la figure 8 un enregistrement qui a donné des résultats favorables.

Sur cet enregistrement, le début de la décompression de la caverne est marqué par une rupture significative de la pente de la courbe représentant l'évolution de l'inclinaison de la surface du sol enregistrée sur le plot $n^{\circ} 3$ (point A). Tous les enregistrements n'ont pas été aussi nets.

Si on fait confiance à celui-ci, le module global des terrains mesuré par l'essai serait assez élevé (15 $000 \mathrm{MPa})$, ce qui peut paraître cohérent avec les données géotechniques: la cavité, située dans la cou-

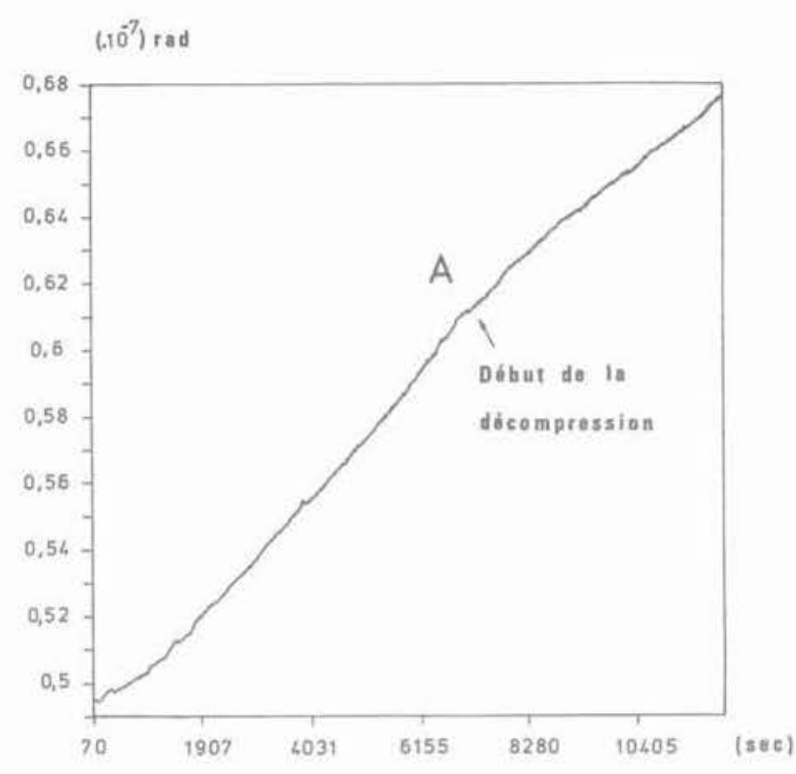

Fig. 8. - Inclinaisons pendant une compressiondécompression.

Fig. 8. - Observed dips during cavern loading and unloading.

che "basse» de sel, est surmontée de six cents mètres de terrains à forte prédominance de sel gemme, matériau assez raide et surtout en général marqué par Pabsence de discontinuités ouvertes.

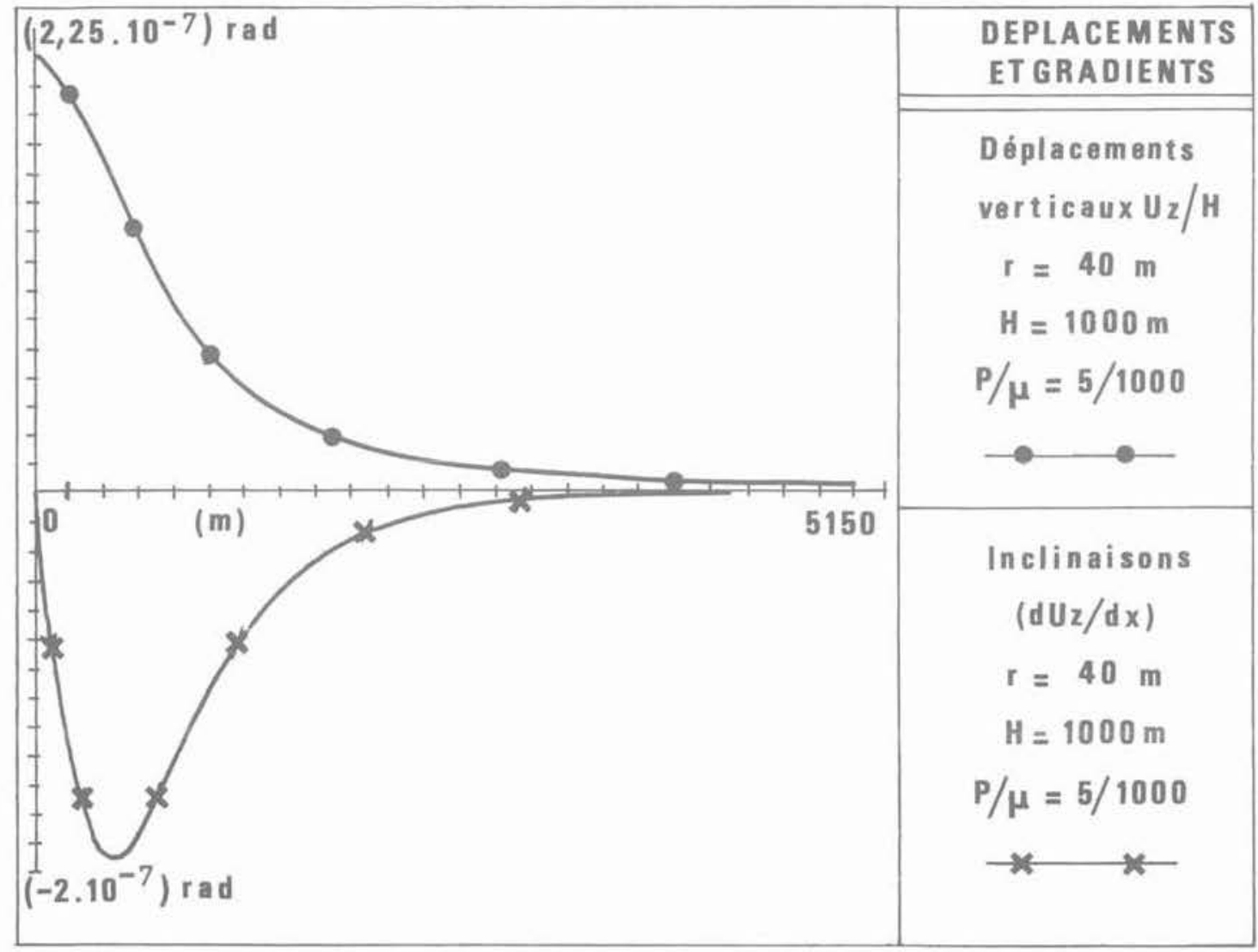

Fig. 9. - Déplacements et inclinaisons calculées à la surface du sol. Fig. 9. - Computed displacements and dips at ground level. 
Il est donc vraisemblable que l'essai ait été conduit dans des conditions concrètes proches des limites de sensibilité de la méthode. La formule donnée plus haut indique que l'intensité du signal observable en surface varie comme la raideur des terrains et le cube de la profondeur: la méthode est donc prometteuse pour des cavités moins profondes réalisées dans des terrains moins raides à grande échelle qu'un massif de sel gemme.

\section{CONCLUSION}

Le kilomètre cube paraît constituer la limite supérieure de l'échelle à laquelle la méthode de mesure proposée peut être efficacement mise en œuvre. L'échelle du décamètre cube (effet d'une surcharge superficielle) est par contre très bien adaptée; une nouvelle campagne de mesure centrée sur cet objectif est en cours. On peut en espérer la mise au point d'une méthode de détermination du module en place des terrains, commode à mettre en cuvre sur chantier, peu coûteuse et susceptible d'apporter un complément précieux aux méthodes plus locales généralement utilisées.

Les auteurs remercient MM. HUGOUT et DURAND, du Département Réservoirs Souterrains (GDF) et la Direction et les personnels de l'Explotation d'Etrez du GDF, notamment $M$. DUPUIS et M. CHARVET, dont l'efficacité et la compétence ont permis la conduite des essais décrits. Ils remercient aussi M.F. ESNOULT et M. VERHILLE de l'IPGP qui ont installé et vérifié l'instrumentation.

\section{BIBLIOGRAPHIE}

ASTE J.P. et al. (1986), «Utilisation d'un clinomètre à très haute résolution pour l'étude du comportement des ouvrages de génie civil». Revue Française de Géotechnique, $n^{\circ} 34$, Paris, janvier 1986, pp. 57-67.

BLUM P.A. (1963), “Contribution à l'étude de la variation de la verticale en un lieun. Annales Géophysiques, tome 19, p. 215-243.

BLUM P.A., GAULON R. (1971), "Détection et traitement des ondes sismiques de très basses fré. quences». Annales Géophysiques, tome 27, pp. 123-140.

BRACE W.F. (1980), "Permeability of crystalline and argilaceous rocks ». International Journal of Rock Mechanics and Mining Science, vol. 17, $\mathrm{n}^{\circ} 5$, Exeter U.K., octobre 1980, p. 241-251,

HETUIN E. (1988), «Influence d'une cavité sphérique dans un massif élastique semi-infini». Rapport interne Laboratoire de Mécanique des Solides, Ecole Polytechnique, Palaiseau, France, octobre 1988.

MANDEL J. (1966), «Annexe XV: Compléments d'élasticité à trois dimensions». Cours de mécanique des milieux continus, tome II, $1^{\text {re }}$ édition Gauthier-Villars éditeur, Paris, 1966, p. 625-626.

MULLER L., EGGER P. (1982), "Standsicherheit und Bau von Kavernen in Abhüngigkeit von den Baugeologischen Bedingungen *. Mai 1982, ISRM Symposium, Aachen. 